\title{
Effect of percutaneous assisted approach on functional rehabilitation for total hip replacement compared to anterolateral approach: study protocol for a randomized controlled trial
}

\author{
Claudia Hendrickx¹, Willem De Hertogh ${ }^{1 *}$, Ulrike Van Daele ${ }^{1}$, Peter Mertens ${ }^{2}$ and Gaetane Stassijns ${ }^{1,3}$
}

\begin{abstract}
Background: The anterolateral approach is a commonly used technique for total hip replacement. It requires the detachment of a large part of the gluteus medius muscle. However, it is known that this muscle has a great impact on hip stability. Using the percutaneous assisted approach the damage to the gluteus medius can be limited. The purpose of this study is to compare the effect of the percutaneous assisted approach with the anterolateral approach on postoperative functional outcome.

Methods/Design: This study uses a prospective, randomized, parallel-group design with blinded assessment and unblinded treatment to compare the percutaneous assisted approach with the anterolateral approach in total hip replacement surgery. The postoperative results of patients operated on using the percutaneous assisted approach will be compared with those of patients operated on using the anterolateral approach. Prior to surgery patients will undergo baseline measurements. These will consist of gluteus medius measurements (surface-electromyography, strength measurements of abductors and quadriceps and the Trendelenburg test), questionnaires (Oxford Hip Score and 36-item Short Form Health Survey) and functional measures (the Timed Get-Up-and-Go test, Five times Sit-to-Stand test and Six-Minute Walk test). These measurements will be repeated four and 12 weeks after surgery. After surgery both groups will receive usual care.
\end{abstract}

Discussion: The gluteus medius is the main stabilizer of the hip joint. Therefore, we assume that functional outcome and gluteus medius function of patients after the percutaneous assisted approach will be better than after the anterolateral approach.

Trial registration: This trial was registered with ClinicalTrials.gov on 8 January 2014, registration number: NCT02032017.

Keywords: Total hip replacement, Approach, Gluteus medius, Rehabilitation, Functional outcome

\section{Background}

Total hip replacement (THR) is one of the most common orthopedic operations. Based on implant sales figures in the Benelux region of Europe (Belgium, The Netherlands and Luxembourg), the number of THR surgeries performed in 2001 has been estimated at 40,000 (1.52 THR per 1,000 inhabitants per year) [1]. Every THR has an estimated cost of $€ 9000$ on average [2] and

\footnotetext{
* Correspondence: willem.dehertogh@uantwerpen.be

${ }^{1}$ Faculty of Medicine and Health Sciences, University of Antwerp, Universiteitsplein 1, 2610 Antwerp, Belgium

Full list of author information is available at the end of the article
}

the length of hospital stay and postoperative care are contributing factors to these costs [2].

Improvements in health-related quality of life (HRQoL) after surgery are reported. However, significant differences between patients and age- and sex-matched controls are noted. An example of this is the scores of the 36-item Short Form Health Survey (SF-36) subscales physical functioning (PF) $(P=0.01)$, role physical $(\mathrm{RP})(P=0.05)$ and vitality $(P=0.05)$ [3]. These lower scores suggest that although patients demonstrate full recovery on psychosocial and pain scales, a deficit in physical functioning remains. This lack of functional recovery has also been found in a 
review by Vissers et al. stating that physical functioning in patients (measured by self-perceived function, clinical measures and actual daily activity) had only recovered to about $80 \%$ of that of controls [4]. Furthermore, Rasch et al. found persisting muscle atrophy two years after surgery in the acting muscles around the hip [5]. This was caused by loss of muscle volume as well as infiltration of fat tissue.

Postoperative outcome can be influenced by the applied surgical procedure. Two approaches are used to perform THR, with the posterior approach being most commonly used in the USA. Leg lengthening and an increased dislocation rate are the downsides [6-8]. A popular technique in Europe is the anterolateral approach (AA). By using this, a large part of the gluteus medius muscle is released to obtain good access to the acetabulum. There are little or no dislocations reported [9], but limping during the first three months is a wellknown problem [6,7].

Muscles are major contributors to hip contact force, with gravitational and centrifugal forces combined contributing less than 5\%. One of the most important muscles for this is the gluteus medius [10]. It is responsible for stabilizing the femoral neck in the acetabulum during the different stages of gait $[11,12]$. This makes it a very important muscle for normal functioning and gait. The gluteus medius is often already weakened before surgery in patients with osteoarthritis of the hip [13]. Since the AA detaches approximately two thirds of the gluteus medius, it affects normal function. Consequently, it can be assumed that interventions sparing the gluteus medius are probably beneficial for patients' postoperative functional outcome.

Damage to the gluteus muscle can be limited using the percutaneous assisted approach (PAA). In this technique, a second small incision $(1 \mathrm{~cm})$ at the anterior border of the femur is made. A canula is placed underneath the muscle and used to pass the reamers in the direction of the acetabulum (Figures 1 and 2). There is no need to enlarge the skin incision or to release more muscle insertion to achieve good working access to the acetabulum. There are two advantages of this procedure: sparing of the gluteus medius muscle and safe access to the acetabulum to obtain perfect positioning of the implants.

Interventions sparing the gluteus medius are probably beneficial for patients' postoperative functional outcome. The purpose of this study is to evaluate the effect of the PAA on postoperative functional outcome compared to the AA.

\section{Methods/Design}

\section{Study design}

This study uses a prospective, randomized, parallel-group design with blinded assessment and unblinded treatment to compare the PAA with the AA in THR surgery.

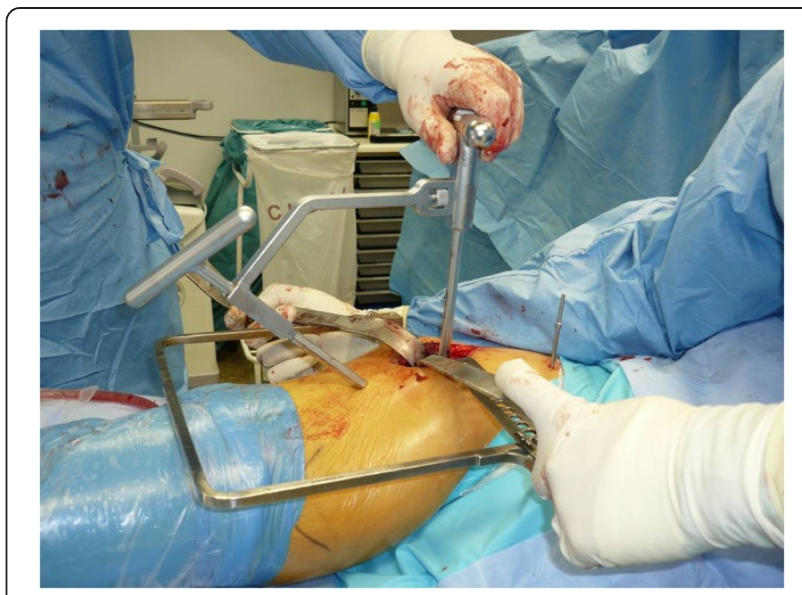

Figure 1 Percutaneous assisted approach, side view.

\section{Ethical approval}

Full ethical approval has been given by the ethics committee of the Antwerp University Hospital (UZA) and Hospital Network Antwerp (ZNA) (approval number: B300201318915).

\section{Patient selection}

Patients will be recruited at the orthopedics department of the ZNA Middelheim hospital in Antwerp, Belgium. Patients with a hip complaint for which THR is indicated are screened for eligibility by a specialized surgeon (PM). Eligible patients will be between 50 and 80 -years-old and suffer from unilateral hip arthritis or avascular necrosis in need of THR. Comorbidities affecting functional outcome will be used as exclusion criteria. These consist of symptomatic lumbar pathology or those in need of surgery or intervention on the ipsilateral knee and/or ankle or foot. Additionally, neurological disorders, according to the

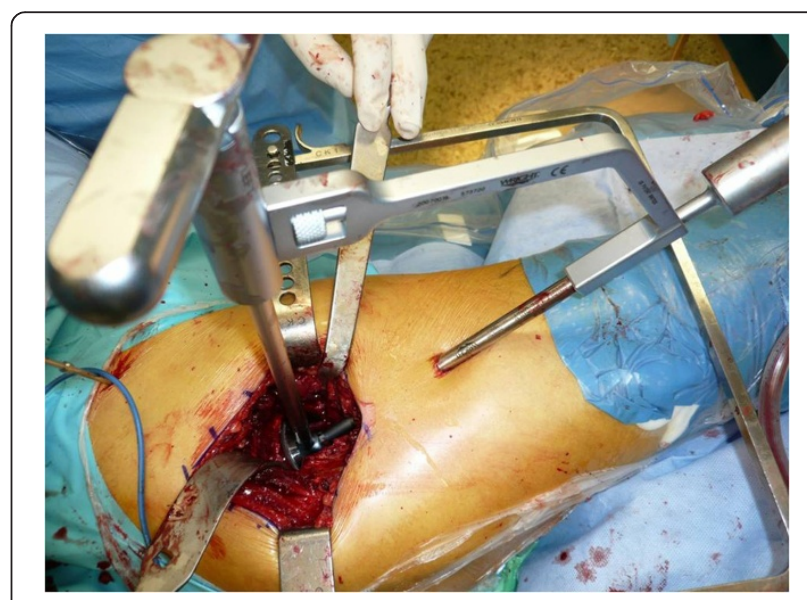

Figure 2 Percutaneous assisted approach, top view. 
International Classification of Diseases, such as Parkinson's disease and previous cardiovascular accidents (CVA) are excluded from this study.

Eligible patients who provide informed consent will be sent to the Antwerp University Hospital (UZA) where an appointment for preoperative (baseline) measurements will be made. These consist of gluteus medius measures, HRQoL-questionnaires and functional measures. Patients will be given an information sheet and a written informed consent will be obtained prior to baseline measurements.

\section{Gluteus medius measures}

\section{Surface-electromyography of gluteus medius muscle}

Patients will perform a single leg stance (SLS) and a maximal voluntary isometric contraction (MVIC) of the gluteus medius during surface-electromyography (SEMG) measurement. Both have shown excellent reliability (SLS intraclass correlation coefficient $(\mathrm{ICC})=0.93$; $\mathrm{MVIC}$ ICC $=$ 0.98 ) [14]. Electrodes will be positioned $2.5 \mathrm{~cm}$ posterior of the middle of the line between the top of the iliac crest and the greater trochanter. This is more posterior than the method of Hermens et al. [15]. This decision is based on a cadaver-study by Van Leemput et al. (Van Leemput W, Collier E, Saeys N, Stassijns G: Measuring tensor fasciae latae and gluteus medius with surface EMG: a cadaver-based study. Unpublished). They demonstrated that the distance from the needle tract to the central point of the muscle was significantly less when the electrodes were placed more posterior $(P<0.0001)$. Therefore we prefer this method.

\section{Quadriceps and abductor muscle strength}

Quadriceps and abductor strength will be measured with the microFET 2 hand-held dynamometer (Biometrics Motion nv, Almere, The Netherlands). Measurements with this device are highly reliable, with excellent intrarater reliability (ICC 0.921 to 0.929 ) and valid, with high correlations with the Timed Get-Up-and-Go test (TGUG) $(r=-0.710$ to -0.862$)$ and gait speed $(r=0.728$ to 0.825$)$ [16].

For quadriceps force the patient is seated with the knees bent $90^{\circ}$. Resistance will be administered on the ventral side of the lower leg, just proximal of the ankle joint. Abductor force will be measured in a supine position. Resistance will be administered on the lateral side of the upper leg, just proximal of the knee joint. Patients will be asked for a MVIC. The tests will be repeated three times. The mean value of the three measurements will be recorded.

\section{Trendelenburg test}

This test measures abductor force in a functional way. The patient is asked to raise one leg (sound side) and raise the non-stance side of the pelvis as high as possible for 30 seconds. The response is classified as followed [17]:

1. Normal: The pelvis on the non-stance side can be lifted maximally for 30 seconds.

2. The pelvis on the non-stance side can be lifted but not maximally

3. The pelvis is elevated but not maintained for 30 seconds

4. No elevating of the pelvis on the non-stance side

5. Drooping of the pelvis

6. Non-valid response: due to hip pain or uncooperative patient

Test-retest reliability coefficients $(\kappa)$ are found to be greater than 0.75 [18].

\section{HRQoL-questionnaires}

All patients will be required to complete the Oxford Hip Score (OHS) and the 36-item Short Form Health Survey (SF-36) questionnaires. The OHS is a disease-specific questionnaire that consists of 12 questions for the evaluation of pain and hip function in relation to various activities. Each question contains five quantifiable answers, leading to a total score that can range from 12 (least problems) to 60 (most problems). For our study population we will use the Dutch version. This version is believed to have excellent test-retest reliability $(\mathrm{ICC}=0.97)$ and a very high correlation with the visual analogue scale score of the hip $(r \geq 0.7)$ [19]. Changes in the OHS are also closely related to the patients' satisfaction with their surgery $[20,21]$. Furthermore, it is very sensitive to change in the first postoperative year [19].

The SF-36 is a generic questionnaire that contains 36 items measuring health on eight different dimensions. These dimensions cover functional status, wellbeing and overall evaluation of health. It draws attention to broader problems of physical functioning than the OHS [22]. The SF-36 is not very sensitive to individual changes, but is effective for measuring group changes, so it becomes a good instrument for scientific research [23]. Internal-consistency reliability of the Dutch version meets the 0.70 level (Cronbach's alpha) recommended for group comparisons on all scales (range: 0.78 to 0.92). The PF and Bodily Pain (BP) scales even met or exceeded the 0.90 level recommended for individual comparisons [24].

\section{Functional measures}

All patients will have functional measurements recorded using the TGUG test, the Five Times Sit-to-Stand test (5tSTS) and the Six-Minute Walk (6MWT) test. In the TGUG test, the participant is asked to stand up and walk $3 \mathrm{~m}$, turn around and walk back to the chair and to 
sit down. The score recorded is the time used to complete the test expressed in seconds [25]. Yeung et al. investigated the psychometric properties of the TGUG test in inpatients on an orthopedic rehabilitation ward [26]. They found a high ICC (0.80). The standard error of measurement was 10.2 seconds. Change in TGUG scores correlated with the changes in pain $(\mathrm{r}=0.21$; $P<0.01)$ and function $(\mathrm{r}=-0.23 ; P<0.01)$.

The 5tSTS test is an easily feasible test where the patient has to stand up and sit back down five times as quickly as possible is a good predictor of falling. A worse score (a longer time needed to complete the test) on the 5tSTS implies a greater chance of falling [27]. Furthermore it predicts problems with daily activities [28]. The test-retest reliability is good to high (ICC 0.64 to .096) in most populations and settings and validity is scored good (compared to 1 Repetition Maximum (1RM) leg press, Pearson correlation coefficient $\mathrm{r}=0.68, P<0.05)$ $[29,30]$. The standard error of measurement $(6.3 \%$ of mean 5tSTS) and the minimum detectable change (17.5\% of mean 5 tSTS) are low [31].

The 6MWT requires a $30 \mathrm{~m}$ hallway, but no exercise equipment or advanced training for technicians. The test measures the distance a patient can quickly walk on a flat, hard surface in a time-period of six minutes (the six-minute walking distance (6MWD)). The test will be carried out according to the American Thoracic Society guidelines [32]. This means there will be a standard instruction and demonstration prior to the test; the researcher is not allowed to walk with the patient. No verbal feedback is given. Every minute the remaining time is mentioned with a standard phrase of encouragement, spoken in a neutral tone. The timer is started when the patient starts walking. The test-retest reliability is excellent (ICC 0.95). The 6MWD is significantly greater for active than for inactive adults $(P<0.0001)$. It moderately correlates with chair stands $(r=0.67)$, standing balance $(r=0.52)$, gait speed $(r=-0.73)$ and self-reported physical functioning $(r=0.55)$ [33].

\section{Intervention}

All patients will get a standard THR (cementless hydroxyapatitecoated cup and a titanium plasma-sprayed stem (Wright Medical, Warshaw, USA)) with a ceramic-on-ceramic (third generation BIOLOX $^{\mathrm{m}}$ delta (Wright Medical, Warshaw, USA) couple. Preoperative leg length and offset are marked to reconstruct the preoperative leg length and to obtain the optimal offset.

In the AA group, a standard transgluteal approach is used and the most optimal component position is achieved. In the PAA group, a muscle sparing technique is used. This will not compromise the component position, but fewer release and detachment of the gluteus medius is necessary. Both groups will receive usual care
(UC) after surgery. This includes standard physiotherapy care consisting of mobilizing and strengthening techniques. All patients will receive a booklet containing information about the surgery, weight-bearing capacity after the surgery and rehabilitation in general.

\section{Follow-up measurements}

All measurements will be repeated at four weeks and three months post-surgery. Six weeks post-surgery patients will be seen by the orthopedic surgeon for clinical and radiographic control. These are routine measurements to assess the postoperative course.

\section{Randomization, blinding and treatment allocation}

Patients will be allocated to one of two treatment groups by the surgeon at the preoperative consultation. Randomization will take place by means of blinded envelopes. In total 10 envelopes are provided: five that allocate the patient to the AA group and five that allocate the patient to the PAA group. This is to ensure that the same number of patients is randomized in one of the two treatment groups. Patients receive the same information from the surgeon regardless of their group allocation. A blinded researcher will perform baseline and follow-up measurements. To ensure blinded assessment, SEMG will be carried out by an independent researcher. This because blinding is not possible due to different locations of scar tissue.

\section{Power and sample size calculation}

Starting with a pilot study, we will include five people in each group. On this basis we will do power and sample size calculations in order to commence with the randomized controlled trial.

\section{Analysis}

The primary outcome measure will be the score on the TGUG. Secondary outcome measures will include SEMG, strength of quadriceps and gluteus medius, OHS and SF-36 questionnaire score, score on the 5tSTS, the Trendelenburg test score and the 6MWT score. Normality of data will be checked via the Kolmogorov-Smirnov test. Differences between both treatment groups at baseline and follow-up measurements will be analyzed using the chi-square statistical method for categorical variables. For continuous variables, comparisons between both groups' baselines will be made using an independent samples t-test. Comparisons between both groups at the follow-up measurements will be made using a twofactor repeated measures Analysis of Variance (ANOVA) (group $\times$ time). Baseline values will be included as the first follow-up measurements. Differences between successive measurements within a treatment group will be analyzed using a one-factor repeated measures ANOVA. 
Significance value for all tests is set at $P<0.05$. The results of all subjects will be analyzed, regardless of their treatment adherence (intention-to-treat analysis).

\section{Discussion}

The aim of this study is to assess the difference in functional outcome after THR through the PAA, compared to THR through the AA. The main difference between these two approaches is their impact on the gluteus medius. Whereas the AA detaches two thirds of the gluteus medius, the PAA only detaches a small part of the muscle. Since the gluteus medius is one of the most important muscles for the stability of the hip joint [10-12], we hypothesize that functional outcome is better or is achieved faster if THR surgery is performed through the PAA instead of the AA.

For this study we chose to have multiple outcome measures (SEMG, strength of gluteus medius and quadriceps, the Trendelenburg test score, the 6MWD and scores on the OHS, SF-36, TGUG and 5tSTS tests) to be certain we measure all possible effects. According to Vissers et al. three aspects should measure functional outcome: self-perceived function, functional tests in a laboratory setting and actual daily activity [4]. Both selfperceived function (measured by the OHS and SF-36 questionnaires) and functional tests (measured by the 6MWT and the TGUG, 5tSTS and tests) are use as outcome measures in our study. However, we chose not to assess actual daily activity at this stage. Rasch et al. reported remaining muscle atrophy up to two years after THR [5]. Therefore, we chose to include muscle strength measurements (measured by the SEMG, MicroFET and Trendelenburg tests). If this protocol takes too much effort for our patients it is possible that we could reduce it by removing the $6 \mathrm{MWT}$ and the Trendelenburg test.

This study has the following limitations. First, complete blinding cannot be achieved because of the SEMG measurements of the gluteus medius and the difference in location of scar tissue for both surgical approaches. Therefore, we chose to have an independent researcher carry out SEMG measurements and note the results, ensuring the other measures will be blinded. Second, we will only evaluate the short-term outcome. Because of the difference in approach we assume that the intervention group will already show a great deal of improvement in the short-term, while the control group will improve less. In later studies it will be necessary to investigate long-term success as well.

The following strengths can be mentioned. First, we conduct a single centre study, ensuring a standardized approach. Second, we use a wide range of outcome measures allowing us to achieve a total picture of the rehabilitation process. This is why patients are asked to complete both a generic (SF-36) and a disease-specific questionnaire (OHS). The disease-specific questionnaire is believed to better measure changes in function. The generic questionnaire is used to detect changes in psychosocial terms as well.

\section{Trial status}

Statistical analysis. Recruitement began October 2013 and was finished in September 2014.

\begin{abstract}
Abbreviations
1RM: One repetition maximum; 5tSTS: Five times Sit-to-Stand; 6MWD: Sixminute walking distance; $6 \mathrm{MWT}$ : Six-Minute Walk test; AA: Anterolateral approach; ANOVA: Analysis of Variance; BP: Bodily Pain; CVA: Cerebrovascular accident; HRQol: Health-related quality of life; MVIC: Maximal voluntary isometric contraction; OHS: Oxford Hip Score; PAA: Percutaneous assisted approach; PF: Physical functioning; RP: Role physical; SEMG: Surfaceelectromyography; SF-36: 36-item Short Form Health Survey; SLS: Single leg stance; TGUG: Timed Get-Up-and-Go; THR: Total hip replacement; UC: Usual care; UZA: Universitair Ziekenhuis Antwerpen (Antwerp University Hospital Antwerp); ZNA: Ziekenhuis Netwerk Antwerpen (Hospital Network Antwerp).
\end{abstract}

\section{Competing interests}

Funding of this study is partially provided by Stöpler Belgium NV.

\section{Authors' contributions}

$\mathrm{CH}$ drafted the manuscript. WDH has been involved in drafting the manuscript, revised it critically for important intellectual content and made substantial contributions to the conception and design of the study. UVD revised the manuscript critically for important intellectual content. PM has been involved in drafting the manuscript, more specifically the technical description of the surgical approaches and the background of the surgical approaches. GS revised the manuscript critically for important intellectual content and made substantial contributions to conception and design of the study. All authors have approved the final manuscript for publication.

\section{Acknowledgments}

This study protocol presents independent research funded by Stöpler Belgium NV and the University of Antwerp under its Dehousse Grant program. The views expressed are those of the authors and not necessarily those of the University of Antwerp or Stöpler Belgium NV.

C. Hendrickx is Dehousse Grant holder.

\section{Author details}

'Faculty of Medicine and Health Sciences, University of Antwerp,

Universiteitsplein 1, 2610 Antwerp, Belgium. ${ }^{2}$ Orthopedic Department, ZNA Middelheim, Lindendreef 1, 2020 Antwerp, Belgium. ${ }^{3}$ Department of Physical Medicine and Rehabilitation, Antwerp University Hospital, Wilrijkstraat 10, 2650 Edegem/Antwerpen, Belgium.

Received: 20 January 2014 Accepted: 17 September 2014

Published: 8 October 2014

\section{References}

1. Scheerlinck TDP, Casteleyn PP: European hip arthroplasty: a survey in university hospitals. Hospital 2003, 5:49-53.

2. De Béthune XBJ, Van Dooren J, Gillet P, Ackaert K: Totale heupprothese in Belgie: Een gedetailleerde analyse van de praktijken en resultaten inzake totale heupprothese: en wat daarna? [Total hip replacement in Belgium: A detailed analysis of practice and results regarding total hip replacement: and what after that?]. CM-informatie 2011, 245:20-24.

3. Nilsdotter AK, Isaksson F: Patient relevant outcome 7 years after total hip replacement for OA - a prospective study. BMC Musculoskelet Disord 2010, 11:47.

4. Vissers MM, Bussmann JB, Verhaar JA, Arends LR, Furlan AD, Reijman M: Recovery of physical functioning after total hip arthroplasty: systematic review and meta-analysis of the literature. Phys Ther 2011, 91:615-629.

5. Rasch A, Bystrom AH, Dalen N, Martinez-Carranza N, Berg HE: Persisting muscle atrophy two years after replacement of the hip. J Bone Joint Surg 2009, 91:583-588. 
6. Edmunds $C T$, Boscainos PJ: Effect of surgical approach for total hip replacement on hip function using Harris Hip scores and Trendelenburg's test: a retrospective analysis. Surgeon 2011, 9:124-129.

7. Ritter MA, Harty LD, Keating ME, Faris PM, Meding JB: A clinical comparison of the anterolateral and posterolateral approaches to the hip. Clin Orthop Relat Res 2001, Apr:95-99.

8. Vicar AJ, Coleman CR: A comparison of the anterolateral, transtrochanteric, and posterior surgical approaches in primary total hip arthroplasty. Clin Orthop Relat Res 1984, Sep:152-159.

9. Roberts JM, Fu FH, McClain EJ, Ferguson AB Jr: A comparison of the posterolateral and anterolateral approaches to total hip arthroplasty. Clin Orthop Relat Res 1984, Jul-Aug:205-210.

10. Correa TA, Crossley KM, Kim HJ, Pandy MG: Contributions of individual muscles to hip joint contact force in normal walking. J Biomech 2010, 43:1618-1622.

11. Al-Hayani A: The functional anatomy of hip abductors. Folia Morphol (Warsz) 2009, 68:98-103.

12. Gottschalk F, Kourosh S, Leveau B: The functional anatomy of tensor fasciae latae and gluteus medius and minimus. J Anat 1989, 166:179-189.

13. Dwyer MK, Stafford K, Mattacola CG, Uhl TL, Giordani M: Comparison of gluteus medius muscle activity during functional tasks in individuals with and without osteoarthritis of the hip joint. Clin Biomech 2013, 28:757-761.

14. Norcross MF, Blackburn JT, Goerger BM: Reliability and interpretation of single leg stance and maximum voluntary isometric contraction methods of electromyography normalization. J Electromyogr Kinesiol 2010, 20:420-425

15. Hermens HJ, Freriks B, Disselhorst-Klug C, Rau G: Development of recommendations for SEMG sensors and sensor placement procedures. J Electromyogr Kinesiol 2000, 10:361-374.

16. Schaubert KL, Bohannon RW: Reliability and validity of three strength measures obtained from community-dwelling elderly persons. J Strength Condit Res 2005, 19:717-720.

17. Hardcastle P, Nade S: The significance of the Trendelenburg test. J Bone Joint Surg 1985, 67:741-746.

18. Roussel NA, Nijs J, Truijen S, Smeuninx L, Stassijns G: Low back pain: clinimetric properties of the Trendelenburg test, active straight leg raise test, and breathing pattern during active straight leg raising. J Manip Physiol Ther 2007, 30:270-278.

19. Gosens T, Hoefnagels NH, de Vet RC, Dhert WJ, van Langelaan EJ, Bulstra SK, Geesink RG: The "Oxford Heup Score": the translation and validation of a questionnaire into Dutch to evaluate the results of total hip arthroplasty. Acta Orthop 2005, 76:204-211.

20. Arden NK, Kiran A, Judge A, Biant LC, Javaid MK, Murray DW, Carr AJ, Cooper C, Field RE: What is a good patient reported outcome after total hip replacement? Osteoarthritis Cartilage 2011, 19:155-162.

21. Fitzpatrick R, Morris R, Hajat S, Reeves B, Murray DW, Hannen D, Rigge M, Williams O, Gregg P: The value of short and simple measures to assess outcomes for patients of total hip replacement surgery. Qual Health Care 2000, 9:146-150.

22. Dawson J, Fitzpatrick R, Murray D, Carr A: Comparison of measures to assess outcomes in total hip replacement surgery. Qual Health Care 1996, 5:81-88.

23. Busija L, Osborne RH, Nilsdotter A, Buchbinder R, Roos EM: Magnitude and meaningfulness of change in SF-36 scores in four types of orthopedic surgery. Health Qual Life Out 2008, 6:55.

24. Aaronson NK, Muller M, Cohen PD, Essink-Bot ML, Fekkes M, Sanderman R, Sprangers MA, te Velde A, Verrips E: Translation, validation, and norming of the Dutch language version of the SF-36 health survey in community and chronic disease populations. J Clin Epidemiol 1998, 51:1055-1068.

25. Podsiadlo D, Richardson S: The timed "Up \& Go": a test of basic functional mobility for frail elderly persons. J Am Geriatr Soc 1991, 39:142-148.

26. Yeung TS, Wessel J, Stratford PW, MacDermid JC: The timed up and go test for use on an inpatient orthopaedic rehabilitation ward. J Orthop Sports Phys Ther 2008, 38:410-417.

27. Tiedemann A, Shimada H, Sherrington C, Murray S, Lord S: The comparative ability of eight functional mobility tests for predicting falls in community-dwelling older people. Age Ageing 2008, 37:430-435.

28. Zhang F, Ferrucci L, Culham E, Metter EJ, Guralnik J, Deshpande N: Performance on five times sit-to-stand task as a predictor of subsequent falls and disability in older persons. J Aging Health 2013, 25:478-492.
29. Bohannon RW: Test-retest reliability of the five-repetition sit-to-stand test: a systematic review of the literature involving adults. J Strength Condit Res 2011, 25:3205-3207.

30. Ritchie C, Trost SG, Brown W, Armit C: Reliability and validity of physical fitness field tests for adults aged 55 to 70 years. J Sci Med Sport 2005, 8:61-70.

31. Goldberg A: The five-times-sit-to-stand-test (FTSST), the short version of the activities-specific balance confidence $(A B C)$ scale, and fear of falling predict step execution time (SET) in older adults. Arch Gerontol Geriatr 2012, 54:434-438.

32. Laboratories ATSCoPSfCPF: ATS statement: guidelines for the six-minute walk test. Am J Respir Crit Care Med 2002, 166:111-117.

33. Harada ND, Chiu V, Stewart AL: Mobility-related function in older adults: assessment with a 6-minute walk test. Arch Phys Med Rehabil 1999, 80:837-841.

doi:10.1186/1745-6215-15-392

Cite this article as: Hendrickx et al:: Effect of percutaneous assisted approach on functional rehabilitation for total hip replacement compared to anterolateral approach: study protocol for a randomized controlled trial. Trials 2014 15:392.

\section{Submit your next manuscript to BioMed Central and take full advantage of:}

- Convenient online submission

- Thorough peer review

- No space constraints or color figure charges

- Immediate publication on acceptance

- Inclusion in PubMed, CAS, Scopus and Google Scholar

- Research which is freely available for redistribution 